\title{
The ROX index as a predictor of standard oxygen therapy outcomes in thoracic trauma
}

\author{
Adrien Cornillon', Juliette Balbo', Julien Coffinet ${ }^{1}$, Thierry Floch², Mathieu Bard ${ }^{2,3}$, Guillaume Giordano-Orsini ${ }^{3,4}$, \\ Jean-Marc Malinovsky ${ }^{1,3}$, Lukshe Kanagaratnam ${ }^{3,5}$, Daphne Michelet ${ }^{1}$ and Vincent Legros ${ }^{2^{*}}$ (D)
}

\begin{abstract}
Background: Thoracic trauma is commonplace and accounts for $50-70 \%$ of the injuries found in severe trauma. Little information is available in the literature as to timing of endotracheal intubation. The main objective of this study was to assess the accuracy of the ROX index in predicting successful standard oxygen (SO) therapy outcomes, and in pre-empting intubation.

Methods: Patient selection included all thoracic trauma patients treated with standard oxygen who were admitted to a Level I trauma center between January 1, 2013 and April 30, 2020. Successful standard SO outcomes were defined as non-requirement of invasive mechanical ventilation within the 7 first days after thoracic trauma.

Results: One hundred seventy one patients were studied, 49 of whom required endotracheal intubation for acute respiratory distress (28.6\%). A ROX index score $\leq 12.85$ yielded an area under the ROC curve of 0.88 with a $95 \% \mathrm{Cl}$ [0.80-0.94], 81.63sensitivity, 95\%Cl [0.69-0.91] and 88.52 specificity, 95\%Cl [0.82-0.94] involving a Youden index of 0.70. Patients with a median ROX index greater than 12.85 within the initial $24 \mathrm{~h}$ were less likely to require mechanical ventilation within the initial 7 days of thoracic trauma.

Conclusion: We have shown that a ROX index greater than 12.85 at $24 \mathrm{~h}$ was linked to successful standard oxygen therapy outcomes in critical thoracic trauma patients. It is our belief that an early low ROX index in the initial phase of trauma should heighten vigilance on the part of the attending intensivist, who has a duty to optimize management.
\end{abstract}

Keywords: Standard oxygen therapy, ROX index, Thoracic trauma, Outcome

\section{Background}

Thoracic trauma (TT) is common place and accounts for $50-70 \%$ of the injuries found in severe trauma $[1,2]$. The anatomical lesions arising from TT, such as pulmonary contusions, rib fractures, pneumothorax and hemothorax, are frequently manifold. TT is more commonly found in association with other traumatic injuries than in isolation [3].

\footnotetext{
* Correspondence: vlegros@chu-reims.fr

${ }^{2}$ Surgical and Trauma Intensive Care Unit, Reims University Hospital, 45 rue Cognacq Jay, 51092 Reims Cedex, France

Full list of author information is available at the end of the article
}

Thoracic trauma goes hand in hand with significant morbidity and mortality in the intensive care unit (ICU).

Several scoring systems are useful for defining TT severity and include the injury severity score (ISS), the abbreviated injury scale (AIS) with particular focus on the thoracic region, and the thoracic trauma severity score (TTSS). These scores can also benefit clinicians as a means of predicting the risk of mechanical ventilation in TT patients [1, 4-6].

Various oxygenation modalities are available to intensive care unit (ICU) clinicians: conventional oxygen therapy via a nasal cannula, full-face and high concentration

(c) The Author(s). 2021 Open Access This article is licensed under a Creative Commons Attribution 4.0 International License, which permits use, sharing, adaptation, distribution and reproduction in any medium or format, as long as you give appropriate credit to the original author(s) and the source, provide a link to the Creative Commons licence, and indicate if changes were made. The images or other third party material in this article are included in the article's Creative Commons licence, unless indicated otherwise in a credit line to the material. If material is not included in the article's Creative Commons licence and your intended use is not permitted by statutory regulation or exceeds the permitted use, you will need to obtain permission directly from the copyright holder. To view a copy of this licence, visit http://creativecommons.org/licenses/by/4.0/ The Creative Commons Public Domain Dedication waiver (http://creativecommons.org/publicdomain/zero/1.0/) applies to the data made available in this article, unless otherwise stated in a credit line to the data. 
oxygen masks (SO), non-invasive ventilation (NIV), or high-flow nasal cannula (HFNC); and invasive mechanical ventilation (MV) [4-6].

Some studies have demonstrated the advantages of HFNC over conventional oxygen therapy in acute respiratory failure. HFNC enhances comfort, oxygenation and respiratory rates [7].

Selecting the best non-invasive oxygen therapy when managing TT remains a challenging task. In any event there should be no delay in administering invasive ventilation, otherwise an increase in ICU morbidity and mortality would prove inevitable [8]. Requirement of invasive MV must be foreseen by clinical criteria $[2,9]$.

There are no simple clinical criteria that can be used at the bedside to predict the respiratory evolution in TT patients. The ROX index, defined as: $\left(\begin{array}{c}S p O 2 \\ F i O 2 \\ R R\end{array}\right)$, was elaborated by Roca et al. $[10,11]$. The ROX index predicts HFNC failure in patients suffering from uniquely pneumonia-related respiratory distress.

The main objective of the present study was to assess the accuracy of the ROX index in predicting successful SO therapy outcomes and in pre-empting intubation in TT patients.

\section{Methods}

\section{Study design}

Admissions to the Surgical and Trauma ICU (Level 1 Trauma Center) at the University Hospital of Reims
(France) were retrospectively analyzed from January 2013 to April 2020. This unit habitually accommodates all patients with suspected or confirmed serious trauma, occurring within a distance of $150 \mathrm{~km}$ in agreement with French trauma network policy [12].

All patients admitted to the ICU for TT (AIS thorax $\geq 1$ ) were screened regardless of oxygen requirements. Patients who received invasive MV on admission and those who were intubated for emergency surgery were excluded (Fig. 1). The ROX index was calculated on admission (H0), then at $2(\mathrm{H} 2), 6(\mathrm{H} 6), 12(\mathrm{H} 12)$ and 24 (H24) hours in all included patients. Nonrequirement of invasive MV was regarded as a successful SO therapy outcome.

\section{Data recording}

Baseline demographic variables were collected including age, sex, trauma mechanism, isolated TT or polytrauma, severity scores (thorax AIS, ISS, SAPS II, TTSS), requirement for and duration of invasive MV, length of critical care unit stay and ICU mortality.

Mode of oxygen therapy delivery to patients (HFNC, $\mathrm{NIV}, \mathrm{SO}$ ) was collected in addition to oxygen saturation as measured by pulse oximetry and respiratory rate (RR).

Inspired fraction of oxygen ( $\mathrm{FiO} 2)$ was collected from ICU nursing charts that showed continuous hourly recording of HFNC and NIV. In the context of SO therapy, Fi02 was calculated in line with the Sequential Organ Failure Assessment formula [13] $(20 \%+(4$ x

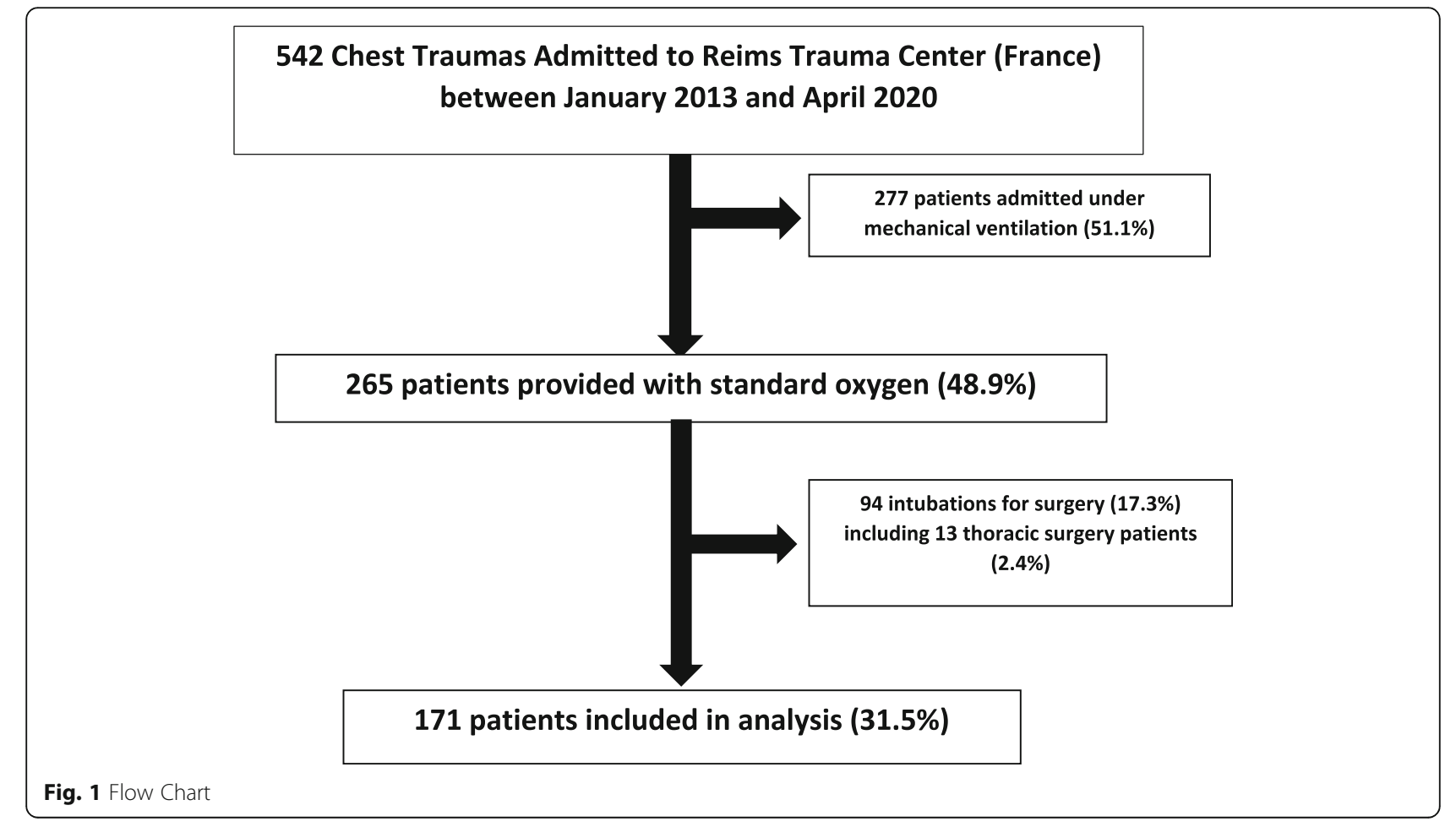


oxygen liter flow per minute), regardless of the interface used (nasal cannula, mask, or high concentration mask).

Intubation indication (surgery or acute respiratory failure) was also collected.

As were manifestation of acute respiratory distress syndrome (ARDS) consistent with the Berlin definition [13], nitric oxide administration, prone position [14] and patient requirement for veno-venous extracorporeal membrane oxygenation (VV-ECMO).

These data were used to calculate the ROX index (defined as the ratio of oxygen saturation as measured by pulse oximetry/FiO2 to respiratory rate) at $\mathrm{H} 0, \mathrm{H} 2, \mathrm{H} 6$, $\mathrm{H} 12$ and $\mathrm{H} 24$.

Multimodal analgesia was administered, including tier 1 analgesics and opioids (mainly via a patient-controlled morphine pump). Pain was assessed several times daily using the visual analog scale. Regional analgesia was delivered insofar as the patient was able to be moved and had no contraindications.

\section{Ethical considerations}

This study was conducted in accordance with the Declaration of Helsinki and approved by the institutional review board (IRB) of the French Society of Anesthesiology and Critical Care (SFAR) (Approval number: IRB 00010254-- 2020-- 09).

STROBE (Strengthening the Reporting of Observational Studies in Epidemiology) guidelines were thoroughly followed [15]. (supplementary material).

\section{Statistical analysis}

Quantitative variables were expressed as medians and interquartile range, categorical variables as frequency and percentages. Continuous variables were compared using the Student's t-test or Mann-Whitney U test, as appropriate. Divergence across categorical variables was assessed via the chi-square or Fisher's exact test, as appropriate. A receiver operating characteristic (ROC) curve was produced and optimal cut-off was defined by the Youden index.

Survival curves regarding higher and lower ROX index values were plotted using the Kaplan Meier method at 2, 4 and 7 days. The log-rank test was used for comparison of these curves.

Cox proportional hazards modeling was used to substantiate any association between the ROX index and requirement for invasive MV. Severity scores (SAPS II, ISS, AIS and TTSS) were additionally applied for adjustment.

Multivariate analysis by logistic regression was conducted to analyze the effect of ROX Index $<12.85$ on SO therapy failure with adjustment for TTSS, simplified acute physiology score II (SAPS II) findings and chronic respiratory disease.
Inclusion of 170 patients was mandatory (to attain 50 SO failures) for sample size calculation based on multivariate analysis involving 4 variables.

Missing data was regarded as missing at random (MAR) and processed by mean imputation.

All analysis was performed using SAS, version 9.4 (SAS Institute, Inc., Cary, NC, USA) and R version 3.6.1 software.

\section{Results}

General characteristics of the included population

Between January 2013 and April 2020, 542 thoracic traumas were admitted to the Level 1 Trauma Center of the University Hospital of Reims (France). 277 patients (51.1\%) were provided with invasive MV on admission. Of the remaining 265 patients (48.9\%), 94 (17.3\%) were placed on invasive MV for emergency surgery, which involved thoracic surgery in 13 (2.4\%) patients (Fig. 1).

171 patients $(31.5 \%)$ were included in the final analysis. Most of the patients were male $(n=134,78.4 \%)$ with a median age of 48 years [31.1-67]. Road traffic accidents (87 patients [50.8\%]) accounted for the most common trauma mechanism. Isolated TT was identified in only 38 cases $(22.2 \%)$, and thoracic intercostal under water sealed drainage was required in 70 cases (40.9\%). Median traumatic severity scores were 20 [13-25], 4 [3, 4], 8 [8-11] for ISS, AIS thorax and TTSS respectively.

49 patients $(28.6 \%)$ required endotracheal intubation for acute respiratory distress within a mean time interval of $33 \mathrm{~h}(-/+53)$ following ICU admission. ICU length of stay was 4 [2-9] days, and 9 patients died on the ICU (5.3\%) (Table 1).

\section{Variables associated with SO failure and ROX index}

The ROX index was significantly lower at different collection times $(\mathrm{H} 0, \mathrm{H} 2, \mathrm{H} 6, \mathrm{H} 12, \mathrm{H} 24)$ in the failed $\mathrm{SO}$ therapy group in which a significantly divergent median ROX per $24 \mathrm{~h}$ of 8.4 [4.9-11.7] vs. 17.8 [14.4-22.1], $p<0.0001$ was observed.

A link was established between age and SO therapy failure (47 years [28.6-63.8] vs. 56.7 years [41.2-72], $p=$ 0.02). Significant correlation was also found between $\mathrm{SO}$ therapy failure and severity scores regarding both trauma and overall severity scores. Treatment by thoracic drainage tube was more commonly required in the failure group $(n=34,69.4 \%)$ vs. successful outcome group ( $n=36,29.5 \%), p<0.0001$.

ICU mortality was higher in the group that received MV: 8 (16.3\%) vs. 1 (0.82\%), $\mathrm{p}<0.0001$ (Table 2).

\section{ROC curve}

A ROC curve was plotted before selecting ROX index cut-offs that made a more accurate prediction of $\mathrm{SO}$ 
Table 1 Baseline Characteristics

\begin{tabular}{ll}
\hline & $\mathbf{N}=\mathbf{1 7 1}$ \\
\hline Age (years) & $48[31.1-67]$ \\
Sex, male, $\mathrm{n}(\%)$ & $134(78.4)$ \\
Chronic respiratory disease, $\mathrm{n}(\%)$ & $12(7)$ \\
Mechanism of Trauma, $\mathrm{n}(\%)$ & \\
$\quad$ Road traffic accident & $87(50.8)$ \\
$\quad$ Hit by object & $16(9.3)$ \\
Fall/jump & $62(36.2)$ \\
$\quad$ Assault & $6(3.5)$ \\
Penetrating trauma, $\mathrm{n}(\%)$ & $6(3.5)$ \\
Isolated chest trauma, $\mathrm{n}(\%)$ & $38(22.2)$ \\
Thoracic intercostal under water sealed drain n (\%) & $70(40.9)$ \\
Injury severity score (ISS) & $20[13-25]$ \\
Abbreviated injury scale (AIS) thorax & $4[3-4]$ \\
Thoracic trauma severity score (TTSS) & $8[5-11]$ \\
Simplified acute physiology score II (SAPS II) & $21[13-33]$ \\
Acute respiratory failure, $\mathrm{n}$ (\%) & $49(28.6)$ \\
Time to intubation (hours) & $33 \pm 53$ \\
Acute respiratory distress syndrome (ARDS), n (\%) & $24(14)$ \\
Prone positioning, $\mathrm{n}$ (\%) & $8(4.7)$ \\
Nitric oxide, $\mathrm{n}$ (\%) & $1(0.6)$ \\
Extracorporeal membrane oxygenation, $\mathrm{n}(\%)$ & $2(1.2)$ \\
Length of ICU stay (days) & $4[2-9]$ \\
ICU deaths, $\mathrm{n}$ (\%) & $9(5.3)$ \\
\hline
\end{tabular}

Data are expressed as medians [interquartile], counts (percentage) or means \pm standard derivation

therapy failure. The area under the ROC curve was 0.88 with a $95 \%$ CI $[0.80-0.94]$.

A ROX index cut-off value of 12.85 yields sensitivity at $81.63,95 \% \mathrm{CI}$ [0.69-0.91], specificity at $88.52,95 \% \mathrm{CI}$ [0.82-0.94], a positive prospective value of $74.07 \%$ [0.62-0.85], a negative predictive value of 92.3\% [0.870.97] and a Youden index of 0.70 (Fig. 2).

\section{Survival analysis}

Kaplan-Meier curves at days 2, 4 and 7 consistent with a ROX index cut-off value of 12.85 are shown in Fig. 3.

MV was significantly more frequent in patients with a ROX index of less than 12.85 ( $p<0.0001$ ) (Fig. 3).

A Cox proportional hazards model was applied to substantiate a relationship between the ROX index of thoracic trauma patients on SO therapy and the risk of MV requirement.

Patients with a mean ROX index greater than 12.85 within the initial $24 \mathrm{~h}$ had less requirement for MV in the first 7 days post thoracic trauma, even after adjustment for various trauma severity scores (ISS, AIS thorax,
TTSS) including adjustment for overall severity scores (SAPS II) (Table 3).

\section{Multivariate analysis}

Multivariate analysis performed on ROX index $<12.85$ adjusted for age, TTSS, SAPS II, and IRC found an OR of 9.86 95\% CI (3.31-29.40) $p<0.001$ (Table 4).

\section{Discussion}

This study reports the first diagnostic performances of the ROX index in predicting successful SO therapy outcomes in TT patients on the ICU. The research work focused on a Level I Trauma Center, reinforcing our cohort which in all probability reflects the most severe cases of thoracic trauma in whom using the ROX index makes sense. A ROX index $\geq 12.85$ at $24 \mathrm{~h}$ was predictive of a successful SO therapy outcome. This threshold remained significant even after adjustment involving the various severity scores (AIS thorax, ISS, TTSS, and SAPS II) at day 2, day 4 and day 7 .

This threshold is much higher than the one initially suggested by Roca et al. ( $\geq 4.88$ at hour 12). A possible explanation for such discrepancy is the type of patients involved, which was significantly different in our retrospective cohort. The present study was based on the assessment of a large number of patients who had sustained thoracic trauma. Most of these patients were young, male and had no respiratory comorbidity. In stark contrast to Roca et al. (35\% of the patients suffered from chronic respiratory insufficiency) [11].

Our populations were likely better able to tolerate their thoracic injuries on account of their healthy pulmonary parenchyma. Additionally, analgesia - one of the priorities when managing TT - could account for a higher ROX index. Few patients received locoregional analgesia partly due to severe polytrauma-induced coagulopathy $[16,17]$. This may have resulted in morphine consumption, induced respiratory-depressing effects and caused a decrease in respiratory rate, leading to an increased ROX threshold in TT patients. With a view to ruling out this bias, it would be worthwhile to conduct a study that has a similar analgesia protocol but includes regional anesthesia to obtain data on opioid dosage.

A higher ROX index could suggest TT patients require faster invasive MV than patients suffering from pneumonia alone. One of the reasons for this may reside in the pain and accompanying damage endured, all of which are relevant factors for clinicians involved in intubation decision-making.

In common with Roca, we produced ROX cut-off points for TT with high specificity, facilitating early identification of patients prone to risk of standard oxygenation failure. 
Table 2 Standard Oxygen Outcomes

\begin{tabular}{|c|c|c|c|}
\hline & Success & $\begin{array}{l}\text { Failure } \\
(n=122)\end{array}$ & $\begin{array}{l}p \text { value } \\
(n=49)\end{array}$ \\
\hline Age (years) & $47[28.6-63.8]$ & $56.7[41.2-72]$ & 0.02 \\
\hline Sex, male, n (\%) & $95(77.8)$ & $39(79.6)$ & 0.8 \\
\hline Chronic respiratory disease, $\mathrm{n}(\%)$ & $6(4.9)$ & $6(12.2)$ & 0.08 \\
\hline Isolated chest trauma, n (\%) & $26(21.3)$ & $12(24.5)$ & 0.6 \\
\hline Thoracic intercostal under water sealed drain n (\%) & $36(29.5)$ & $34(69.4)$ & $<0.0001$ \\
\hline \multicolumn{4}{|l|}{ Severity score: } \\
\hline- ISS & 17 [13-24] & 20 [16-29] & 0.0001 \\
\hline - AlS Thorax & $3[3-4]$ & $4[4-4]$ & 0.003 \\
\hline - TTSS & $8[4-10]$ & $12[9-14]$ & $<0.0001$ \\
\hline - SAPS ॥ & $17[11-25]$ & 34 [16-29] & $<0.0001$ \\
\hline HFNC required in initial 24 h, $\mathrm{n}(\%)$ & $0(0)$ & $6(12.2)$ & $<0.0001$ \\
\hline NIV required in initial $24 \mathrm{~h}, \mathrm{n}(\%)$ & $2(1.6)$ & $0(0)$ & $<0.0001$ \\
\hline \multicolumn{4}{|l|}{ Rox Index } \\
\hline $\mathrm{HO}$ & 15.5 [11.7-20.8] & 7.3 [4.9-10.6] & $<0.0001$ \\
\hline $\mathrm{H} 2$ & 17.6 [12.9-21.4] & $10.7[5.3-14.8]$ & $<0.0001$ \\
\hline $\mathrm{H} 6$ & 17.8 [14.6-23.1] & 11.1 [7.9-18.5] & 0.001 \\
\hline $\mathrm{H} 12$ & $18.2[15.1-22.8]$ & $2.9[7.8-18.5]$ & 0.0013 \\
\hline $\mathrm{H} 24$ & $18.2[14.9-23.8]$ & 13.6 [7.9-16.6] & 0.0013 \\
\hline Mean/24 h & $17.8[14.4-22.1]$ & 8.4 [4.9-11.7] & $<0.0001$ \\
\hline ICU deaths, n (\%) & $1(0.82)$ & $8(16.3)$ & $<0.0001$ \\
\hline
\end{tabular}

Data are expressed as medians [interquartile] or counts (percentage), HFNC High flow nasal cannula, NIV Non-invasive ventilation

Optimal timing of intubation is a constant cause of concern for clinicians. Late intubation has been linked to poorer outcomes in patients with acute respiratory failure [18]. Invasive MV in TT patients is related to longer ICU stays, pneumonia, infection rates and mortality [19].

Data surrounding invasive MV highlight the complexity of discerning whether or not to intubate a TT patient, especially since strong predictive factors to underpin decision-making are nonexistent. A ROX index $\geq 12.85$ at $24 \mathrm{~h}$ applied to TT cases could be a valuable tool in tackling this challenge.

The ROX index has also been addressed in relation to various management time intervals ( $\mathrm{H} 0, \mathrm{H} 2, \mathrm{H} 6$, $\mathrm{H} 12$, and H24). A significant threshold was observed for each of these intervals. These results suggest that the ROX index could be used at an earlier stage in TT management. It would also be worthwhile to study the applicability of this index in pre-hospital care settings following analgesia, particularly in regions where a nearby trauma center is lacking. Larger prospective studies will be required to confirm these results.

French guidelines recommend using non-invasive ventilation (NIV) in TT and hypoxemic patients [3].
There is a dearth of data assessing HFNC in this indication. NIV likely provides better protection against atelectasis formation that is a common complication of thoracic injury [20]. However, these data require confirmation in a bigger cohort on account of the small patient numbers receiving this type of treatment in our study. Antonelli et al. showed that $\mathrm{PaO} 2 / \mathrm{FiO} 2<146$ within $1 \mathrm{~h}$ of NIV was an independent factor for requiring intubation. This has been demonstrated in patients suffering from acute respiratory distress, $25 \%$ of whom had sustained TT [8]. The fact that so few patients received NIV at $24 \mathrm{~h}$ in this retrospective cohort is unfortunate and restricts the scope of the ROX index.

It is conceivable that a different threshold would come to light in patients treated with NIV. It is our belief that a prospective observational study placing emphasis on the ROX index and NIV would be worthwhile.

In the present study, median time to intubation was $33 \mathrm{~h}+/-53$ and likely stems from the complexity and different mechanisms of TT (parietal lesions, vessel damage, organ injury). This time interval indicates that the condition of TT patients is not always critical straightaway but can deteriorate further down the line. It is highly likely that bronchial obstruction, atelectasis formation and an increase in contusions, weak cough and 


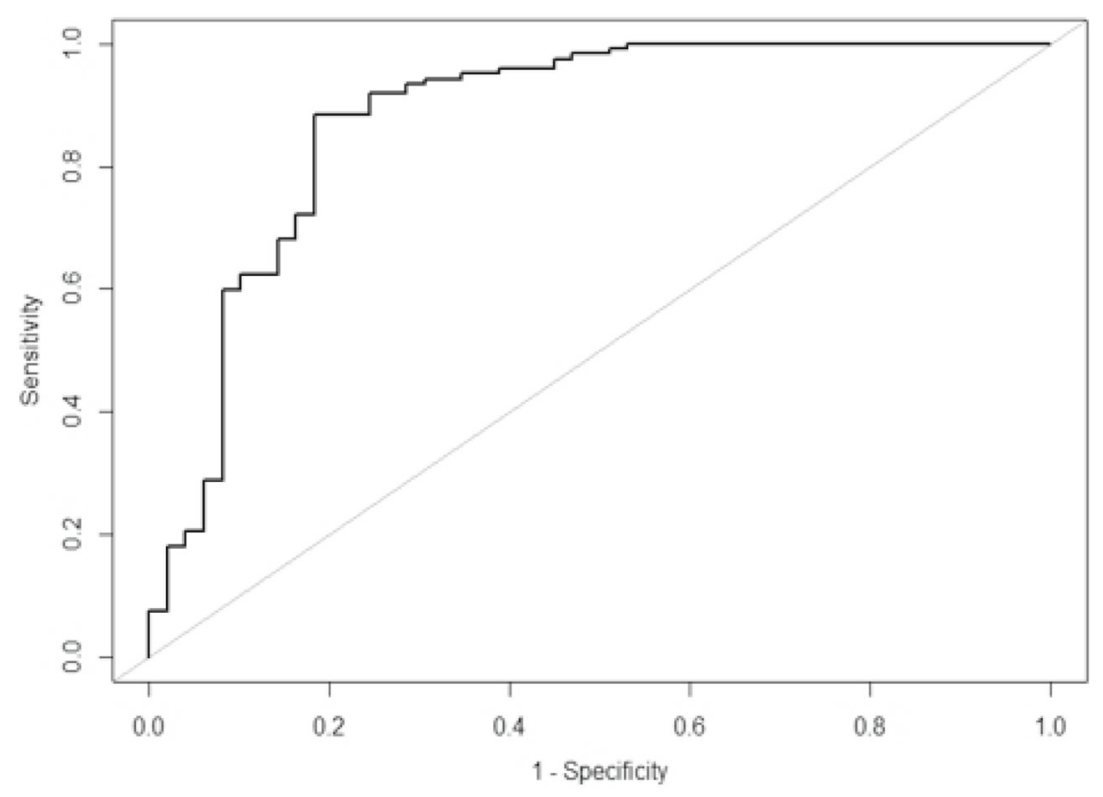

Value

$95 \% \mathrm{CI}$

$\begin{array}{ll}\text { AUC } & 0.88\end{array}$

$0.80-0.94$

Sensitivity

Specificity

Positive predictive value

Negative predictive value
81.63

88.52

74.07

92.3
$0.69-0.91$

$0.82-0.94$

$0.62-0.85$

$0.87-0.97$

Fig. 2 ROC curve for Rox index cut-off of at 12.85. ROC = receiver operating characteristic; $A U C=$ area under curve; $\mathrm{Cl}=$ confidence intervals

respiratory exhaustion accounted for such deterioration. It is noteworthy that mortality was significantly higher in the SO failure group. Several variables such as the clinical severity of the initial injury report (AIS Thorax, ISS, TTSS, SAPS II) could provide an explanation for these findings, but overly late intubation may have aggravated underlying lesions and favored this dimension of poor outcome.

In addition, although patients intubated for neurological coma were excluded, no data was collected regarding brain injuries that potentially aggravate respiratory function.

The three major features of TT typically reported are: contusions, pulmonary wounds and parietal lesions. These different features can each lead to respiratory distress even if the pathophysiology involved varies. Pulmonary contusions result from alveolar-capillary rupture which may develop into pneumonia over several days, entailing significant mortality in intensive care [21, 22]. Lung wounds mainly caused by ballistic or stab wounds present as both open and closed thoracic wounds. They often involve lesions to the large vessels or the intrathoracic organs (heart, aorta, pericardium, pleura) with initial hemorrhagic shock [23, 24]. These patients require urgent surgery, rendering futile any application of the ROX index.

While thoracic wall lesions lead to problems with the mechanics of ventilation, flail chest involving paradoxical breathing is the most severe parietal lesion. It does not strictly speaking lead to respiratory distress but can develop into respiratory exhaustion $[25,26]$.

Few patients were treated surgically for stabilization of costal lesions but were excluded from the study either because they were scheduled for intubation or had already been intubated on admission. Recent studies have explored reducing the duration of MV and length 

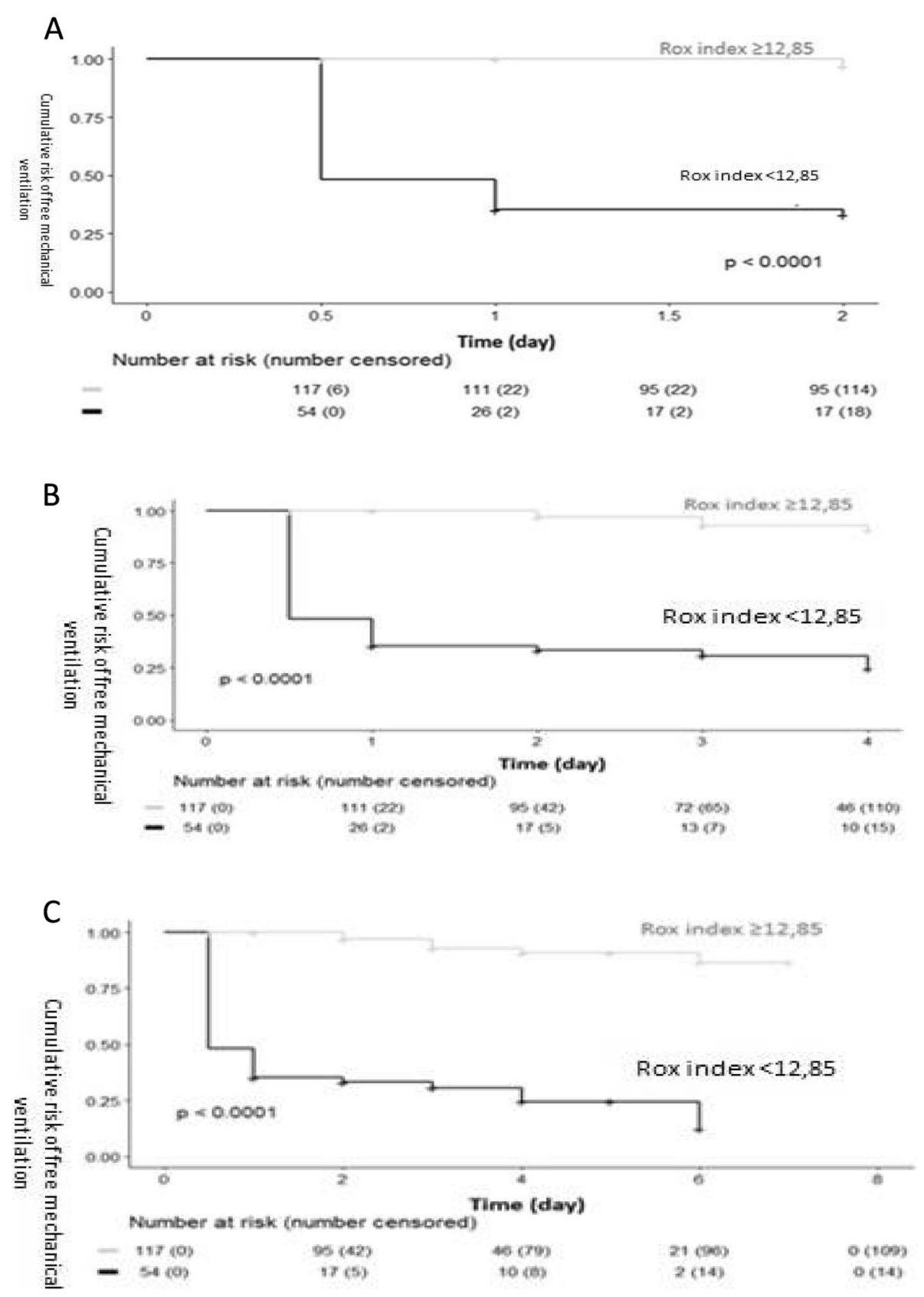

Fig. 3 Kaplan-Meier Curves. Kaplan-Meier curves at Day 2 (A), day (B), and Day 7 (C)

of resuscitation stay in flail chest patients [27, 28]. A French multicenter study is currently underway with the aim of substantiating these encouraging results. Similarly, the respective benefits of early surgical management (within $48 \mathrm{~h}$ ) or later surgical management (5-10 days) are not clearly defined [29-32]. The ROX index could be advanced as a guide for surgical management of isolated TT.

Attention should however be drawn to certain limitations. Firstly, our monocentric retrospective study was conducted over 8 years, during which time there were advances in trauma treatment practice, thus modifying the criteria for MV. Larger multicenter validation studies are needed to establish the role of the ROX index in TT.

Secondly, time of intubation is left to the discretion of the treating clinician in the absence of any formally written protocol based on clinical and biological criteria. A post-hoc analysis was carried out on the basis of patient health record analysis confirming the indication for intubation; the expert estimated that 45 of the 49 intubated patients actually required this procedure. 
Table 3 Cox proportional hazards model (Cox Regression) for analysis of effect of ROX Index $\geq 12.85$ on SO at days 2, 4 and 7 and severity scores

\begin{tabular}{|c|c|c|c|}
\hline & HR & $95 \% \mathrm{Cl}$ & $P$-value \\
\hline \multicolumn{4}{|c|}{ Unadjusted ROX index $\geq 12.85$} \\
\hline At day 2 & 0.03 & $0.009-0.095$ & $<0.0001$ \\
\hline At day 4 & 0.06 & $0.025-0.129$ & $<0.0001$ \\
\hline At day 7 & 0.06 & $0.027-0.129$ & $<0.0001$ \\
\hline \multicolumn{4}{|c|}{ Adjusted by ISS } \\
\hline At day 2 & 0.03 & $0.010-0.106$ & $<0.0001$ \\
\hline At day 4 & 0.06 & $0.034-0.141$ & $<0.0001$ \\
\hline At day 7 & 0.06 & $0.029-0.137$ & $<0.0001$ \\
\hline \multicolumn{4}{|c|}{ Adjusted by AIS thorax } \\
\hline At day 2 & 0.03 & $0.010-0.106$ & $<0.0001$ \\
\hline At day 4 & 0.06 & $0.027-0.139$ & $<0.0001$ \\
\hline At day 7 & 0.06 & $0.029-0.136$ & $<0.0001$ \\
\hline \multicolumn{4}{|c|}{ Adjusted by TTSS } \\
\hline At day 2 & 0.04 & $0.012-0.142$ & $<0.0001$ \\
\hline At day 4 & 0.07 & $0.034-0.187$ & $<0.0001$ \\
\hline At day 7 & 0.08 & $0.036-0.182$ & $<0.0001$ \\
\hline \multicolumn{4}{|c|}{ Adjusted by SAPS II } \\
\hline At day 2 & 0.04 & $0.013-0.146$ & $<0.0001$ \\
\hline At day 4 & 0.08 & $0.034-0.190$ & $<0.0001$ \\
\hline At day 7 & 0.06 & $0.029-0.136$ & $<0.0001$ \\
\hline
\end{tabular}

ISS Injury severity score; AIS Abbreviated injury scale; TTSS Thoracic trauma severity score; SAPS II Simplified acute physiology score II

Thirdly, we are aware that calculation of FiO2 under SO therapy lacks accuracy. As a patient's inspiratory flow rate is higher than the gas flow rate administered by the device, the oxygen administered via the device is mixed with ambient air (FIO2 at 21\%) and the resulting FIO2 cannot be measured. Higher patient rates of tachypnea with high inspiratory flow result in greater variation in the ratio of ambient air to enriched air. In the light of these physical phenomena, $\mathrm{FiO} 2$ percentages in $\mathrm{SO}$ are overestimated. This translates into a similar link between respiratory rate and a lower ROX index which is necessarily a cause of concern for intensivists.

Finally, management of oxygen therapy and mode of administration were governed not by a titration protocol but by a minimum oxygen saturation target based on patient characteristics.

Table 4 Multivariate analysis to substantiate relationship between ROX Index $<12.85$ and SO failure

\begin{tabular}{llll}
\hline & OR & 95\%Cl & $p$-value \\
\hline ROX index $<\mathbf{1 2 . 8 5}$ & 9.869 & $3.313-29.402$ & $<.0001$ \\
\hline
\end{tabular}

Odds ratio adjusted for age, thoracic trauma severity score (TTSS), simplified acute physiology score II (SAPS II) and chronic respiratory disease

\section{Conclusion}

We have shown that a ROX index greater than 12.85 at $24 \mathrm{~h}$ was linked to successful standard oxygen therapy outcomes in critical thoracic trauma patients.

In our opinion, early low ROX index in the initial phase of trauma should heighten vigilance on the part of the attending intensivist, who has a duty to optimize management (thoracic tube insertion, NIV and particular focus on regional anesthesia).

\section{Supplementary Information}

The online version contains supplementary material available at https://doi. org/10.1186/s13049-021-00876-4.

Additional file 1.

Acknowledgements

Dr. Claire LEPOUSE, Dr. Pascal RACLOT, Dr. Marion LECLERCQ-ROUGET, Dr. Elodie GELISSE, From Surgical and Trauma Intensive Care Unit of Reims University Hospital, Reims, France.

\section{Authors' contributions}

$A C, J B, J C, T F, G G O, M B, J M M, D M$, and VL contributed to the conception and study design, analysis and interpretation of data, drafting and revising the manuscript. LK and VL contributed to analysis and interpretation of data. All authors read and approved the final manuscript.

\section{Funding}

None.

\section{Availability of data and materials}

The datasets used and/or analysed during the current study are available from the corresponding author on reasonable request.

\section{Declarations}

Ethics approval and consent to participate

Data analyses are conducted using anonymised data which is governed by a code of practice approved by the French Society of Anesthesiology and Critical Care.

This study was conducted in accordance with the Declaration of Helsinki and approved by the institutional review board (IRB) of the French Society of Anesthesiology and Critical Care (SFAR) (Approval number: IRB $00010254--$ 2020-- 09).

\section{Consent for publication}

Not applicable.

\section{Competing interests}

The authors declare that they have no competing interests.

\section{Author details}

${ }^{1}$ Department of Anesthesiology and critical care, Reims University Hospital, Reims, France. ${ }^{2}$ Surgical and Trauma Intensive Care Unit, Reims University Hospital, 45 rue Cognacq Jay, 51092 Reims Cedex, France. ${ }^{3}$ University of Reims Champagne Ardennes, Reims, France. ${ }^{4}$ Department of Emergency Medicine, Reims University Hospital, Reims, France. ${ }^{5}$ Clinical Research Unit, Reims University Hospital, Reims, France.

Received: 20 January 2021 Accepted: 21 April 2021

Published online: 21 June 2021

\section{References}

1. Probst C, Pape H-C, Hildebrand F, Regel G, Mahlke L, Giannoudis P, et al. 30 years of polytrauma care: an analysis of the change in strategies and results of 4849 cases treated at a single institution. Injury. 2009;40(1):77-83. https:// doi.org/10.1016/j.injury.2008.10.004. 
2. Kulshrestha P, Munshi I, Wait R. Profile of Thoracic Trauma in a Level I Trauma Center. J Trauma Inj Infect Crit Care. 2004;57(3):576-81.

3. Société française d'anesthésie et de réanimation, d'urgence $\mathrm{S}$ française de médecine. Traumatisme thoracique : prise en charge des 48 premières heures. Anesth Réanimation. 2015;1(3):272-87.

4. Mu GH, Lu ZQ, Li X, Chen PF, He L, Sun YH, et al. Profile of thoracic injuries and oxygen therapy in trauma patients with acute respiratory failure after the Jiangsu tornado in China: a retrospective study. Prehospital Disaster Med. 2020;35(3):335-40. https://doi.org/10.1017/S1049023X20000345.

5. Chawla R, Dixit SB, Zirpe KG, Chaudhry D, Khilnani GC, Mehta Y, et al. ISCCM guidelines for the use of non-invasive ventilation in acute respiratory failure in adult ICUs. Indian J Crit Care Med Peer-Rev Off Publ Indian Soc Crit Care Med. 2020;24(Suppl 1):S61-81.

6. Antonelli M, Conti G, Rocco M, Bufi M, De Blasi RA, Vivino G, et al. A comparison of noninvasive positive-pressure ventilation and conventional mechanical ventilation in patients with acute respiratory failure. $N$ Engl J Med. 1998;339(7):429-35.

7. Roca O, Riera J, Torres F, Masclans JR. High-flow oxygen therapy in acute respiratory failure. Respir Care. 2010;55(4):408-13.

8. Antonelli M, Conti G, Moro ML, Esquinas A, Gonzalez-Diaz G, Confalonieri M, et al. Predictors of failure of noninvasive positive pressure ventilation in patients with acute hypoxemic respiratory failure: a multi-center study. Intensive Care Med. 2001;27(11):1718-28. https://doi.org/10.1007/s00134001-1114-4.

9. Bellani G, Laffey JG, Pham T, Fan E, Brochard L, Esteban A, et al. Epidemiology, Patterns of Care, and Mortality for Patients With Acute Respiratory Distress Syndrome in Intensive Care Units in 50 Countries. JAMA. 2016;315(8):788.

10. Roca O, Messika J, Caralt B, García-de-Acilu M, Sztrymf B, Ricard J-D, et al. Predicting success of high-flow nasal cannula in pneumonia patients with hypoxemic respiratory failure: the utility of the ROX index. J Crit Care. 2016; 35:200-5. https://doi.org/10.1016/j.jcrc.2016.05.022.

11. Roca O, Caralt B, Messika J, Samper M, Sztrymf B, Hernández G, et al. An Index Combining Respiratory Rate and Oxygenation to Predict Outcome of Nasal High-Flow Therapy. Am J Respir Crit Care Med. 2019;199(11):1368-76.

12. Hamada SR, Gauss T, Duchateau F-X, Truchot J, Harrois A, Raux M, et al. Evaluation of the performance of French physician-staffed emergency medical service in the triage of major trauma patients. J Trauma Acute Care Surg. 2014;76(6):1476-83. https://doi.org/10.1097/TA.0000000000000239.

13. Definition Task Force ARDS, Ranieri VM, Rubenfeld GD, Thompson BT, Ferguson ND, Caldwell $\mathrm{E}$, et al. Acute respiratory distress syndrome: the Berlin Definition. JAMA. 2012;307(23):2526-33.

14. Guérin C, Reignier J, Richard J-C, Beuret P, Gacouin A, Boulain T, et al. Prone positioning in severe acute respiratory distress syndrome. N Engl J Med. 2013;368(23):2159-68.

15. Vandenbroucke JP, von Elm E, Altman DG, Gøtzsche PC, Mulrow CD, Pocock SJ, et al. Strengthening the Reporting of Observational Studies in Epidemiology (STROBE): explanation and elaboration. PLoS Med. 2007:4(10):e297.

16. Bonet A, Madrazo Z, Koo M, Otero I, Mallol M, Macia I, et al. Thromboelastometric profile and acute coagulopathy of the Polytraumatized patient: clinical and prognostic implications. Cirugia Espanola. 2018:96(1):41-8. https://doi.org/10.1016/j.ciresp.2017.09.012.

17. Sheppard FR, Schaub LJ, Cap AP, Macko AR, Moore HB, Moore EE, et al. Whole blood mitigates the acute coagulopathy of trauma and avoids the coagulopathy of crystalloid resuscitation. J Trauma Acute Care Surg. 2018; 85(6):1055-62.

18. Kangelaris KN, Ware LB, Wang CY, Janz DR, Zhuo H, Matthay MA, et al. Timing of intubation and clinical outcomes in adults with acute respiratory distress syndrome. Crit Care Med. 2016;44(1):120-9. https://doi.org/10.1097/ CCM.0000000000001359

19. Bolliger CT, Van Eeden SF. Treatment of multiple rib fractures. Thoracic. 1990;97(4):943-8.

20. Hernandez G, Fernandez R, Lopez-Reina P, Cuena R, Pedrosa A, Ortiz R, et al. Noninvasive ventilation reduces intubation in thoracic trauma-related hypoxemia: a randomized clinical trial. Thoracic. 2010;137(1):74-80.

21. Bader A, Rahman U, Morris M, McCormack JE, Huang EC, Zawin M, et al. Pulmonary contusions in the elderly after blunt trauma: incidence and outcomes. J Surg Res. 2018;230:110-6. https://doi.org/10.1016/j.jss.2 018.04.049.
22. van Wessem KJP, Hennus MP, van Wagenberg L, Koenderman L, Leenen $\mathrm{LPH}$. Mechanical ventilation increases the inflammatory response induced by lung contusion. J Surg Res. 2013;183(1):377-84. https://doi.org/10.1016/j. jss.2012.12.042.

23. Lichte P, Oberbeck R, Binnebösel M, Wildenauer R, Pape H-C, Kobbe P. A civilian perspective on ballistic trauma and gunshot injuries. Scand J Trauma Resusc Emerg Med. 2010;18:35.

24. Morrison JJ, Mellor A, Midwinter M, Mahoney PF, Clasper JC. Is pre-hospital thoracotomy necessary in the military environment? Injury. 2011;42(5):46973. https://doi.org/10.1016/j.injury.2010.03.009.

25. Tzelepis GE. Thoracic Wall diseases: respiratory pathophysiology. Clin Thoracic Med. 2018;39(2):281-96.

26. Wanek S, Mayberry JC. Blunt thoracic trauma: flail thoracic, pulmonary contusion, and blast injury. Crit Care Clin. 2004;20(1):71-81. https://doi.org/1 0.1016/50749-0704(03)00098-8.

27. Jayle CPM, Allain G, Ingrand P, Laksiri L, Bonnin E, Hajj-Chahine J, et al. Flail thoracic in polytraumatized patients: surgical fixation using Stracos reduces ventilator time and hospital stay. Biomed Res Int. 2015;2015:624723.

28. Doben AR, Eriksson EA, Denlinger CE, Leon SM, Couillard DJ, Fakhry SM, et al. Surgical rib fixation for flail thoracic deformity improves liberation from mechanical ventilation. J Crit Care. 2014;29(1):139-43. https://doi.org/1 0.1016/j.jcrc.2013.08.003.

29. de Moya M, Bramos T, Agarwal S, Fikry K, Janjua S, King DR, et al. Pain as an Indication for Rib Fixation: A Bi-Institutional Pilot Study. J Trauma Inj Infect Crit Care. 2011;71(6):1750-4.

30. Palmer CS, Gabbe BJ, Cameron PA. Defining major trauma using the 2008 abbreviated injury scale. Injury. 2016;47(1):109-15. https://doi.org/10.1016/j. injury.2015.07.003.

31. Aukema TS, Beenen LF, Hietbrink F, Leenen LP. Validation of the thorax trauma severity score for mortality and its value for the development of acute respiratory distress syndrome. Open Access Emerg Med OAEM. 2011; 3:49-53. https://doi.org/10.2147/OAEM.S22802.

32. Pape HC, Remmers D, Rice J, Ebisch M, Krettek C, Tscherne H. Appraisal of early evaluation of blunt thoracic trauma: development of a standardized scoring system for initial clinical decision making. J Trauma. 2000;49(3):496504. https://doi.org/10.1097/00005373-200009000-00018.

\section{Publisher's Note}

Springer Nature remains neutral with regard to jurisdictional claims in published maps and institutional affiliations.

\section{Ready to submit your research? Choose BMC and benefit from:}

- fast, convenient online submission

- thorough peer review by experienced researchers in your field

- rapid publication on acceptance

- support for research data, including large and complex data types

- gold Open Access which fosters wider collaboration and increased citations

- maximum visibility for your research: over $100 \mathrm{M}$ website views per year

At BMC, research is always in progress.

Learn more biomedcentral.com/submissions 